\title{
The Series Bridge Converter (SBC): A Hybrid Modular Multilevel Converter for HVDC Applications
}

\author{
Emmanuel Amankwah*, Alessandro Costabeber*, Alan Watson*, David Trainer ${ }^{\dagger}$, \\ Omar Jasim ${ }^{\dagger}$, Javier Chivite - Zabalza ${ }^{\dagger}$ and Jon Clare* \\ *The University of Nottingham, University Park, Nottingham, NG7 2RD, UK \\ $\dagger$ GE Energy Connections, St Leonard Avenue, Stafford, ST17 4LX, UK \\ Tel.: $+44(0)-1158468840$ \\ E-Mail: Emmanuel.Amankwah@nottingham.ac.uk \\ URL: http://www.nottingham.ac.uk
}

\section{Keywords}

«VSC», «Multilevel Converters», «HVDC», «FACTS», «MMC» «High Voltage Power Converter»

\begin{abstract}
This paper presents a novel hybrid modular multilevel voltage source converter suitable for HVDC applications. It has the advantages of other modular multilevel topologies and can be made more compact making it attractive for offshore stations and city infeed applications. The Operating principle of the converter and internal energy management are discussed with simulation results from a scaled medium voltage demonstrator presented to validate the concepts.
\end{abstract}

\section{Introduction}

Several Modular Multilevel Voltage Source Converters (MM-VSC) have been recently developed for HVDC and FACTS applications [1-4]. These converters offer significant benefits over existing HVDC stations based on Line Commutated Converters (LCC). The operation of MM-VSCs improves the quality of AC current and voltage waveforms while limiting voltage stresses across semiconductor devices. The modular approach makes the converters scalable and adaptable to a wide range of high voltage and high power applications. A number of MM-VSCs are currently in operation and many more are planned [5-7]. Among the others, the Modular Multilevel Converter (MMC) [8] is the focus of many research and industrial projects [9], for its well-known advantages in terms of high power quality, low losses, and ability to inject into weak networks.

In the last decade a number of MM-VSC topologies suitable for HVDC $[1,3,4,10]$ have been proposed in the literature and extensively investigated. Among them, the Alternate Arm Converter (AAC) [3] which is a combination of the original MMC concept and the 2-level converter concept. If the MMC and the AAC are based on a parallel connection of phases on the DC side, another family of converters can be developed based on a series connection configuration on the DC side $[1,11]$.

The Series Bridge Converter (SBC) topology [12] is based on the concept originally proposed in [1]. The converter arrangement uses a series connection on the DC side of three single phase converters, each of them made of a combination of shunt connected half bridge submodules and series connected full bridge submodules. Each phase synthesises a full wave rectified sinusoid which is unfolded by a line frequency soft-switched $\mathrm{H}$-bridge converter to generate the $\mathrm{AC}$ voltage. The converter operation supports both active power exchange and reactive power compensation. With the proposed circuit, the number of submodules required is typically $30-35 \%$ of that required by an equivalent MMC. In this paper, the converter topology and its operating principles are introduced. Energy management within the converter is also discussed. The analysis is validated with detailed simulation results from a medium voltage scaled demonstrator. 


\section{Converter Topology}

Figure 1 shows the basic structure of the SBC. As shown, each phase is composed of two converter arms and an H-bridge. The shunt connected arm with half-bridge submodules is named Chain-Link (CL), while the series connected arm with full-bridge submodules is called Series Full Bridges arm (SFB). The CL and SFB arms are responsible for synthesising a variable amplitude full wave rectified sinusoidal voltage at the DC input of the main H-bridge, that unfolds the waveform at the zero crossings to generate the $\mathrm{AC}$ voltages across the primary side of three open winding transformers as shown in Figure 1. The presence of the transformers is necessary to enable the connection of the converter on the AC side. The three series connected CL units support the DC bus voltage while the three SFB units operate to decouple the AC network from the DC network and achieve full control of the AC side current and voltage.

The resulting converter can be made more compact considering the two main advantages of the topology:

- The series connection on the DC side with a single rail-to-rail chain of submodules supporting the full DC bus, rather than the three in the MMC;

- The unfolding process operated by the main $\mathrm{H}$ bridge guarantees higher frequency AC components of voltages and currents within the submodules and therefore higher frequency voltage ripple across the submodules capacitors.

Another relevant advantage is that only a fraction of the DC current flows through the CL arms, reducing conduction loss. Though the H-bridge based SFB arms are placed in the full current path, the number of submodules in the CL and the SFB arms are optimally selected to achieve the required PQ operation; - reducing significantly the conduction loss and the size of the converter.

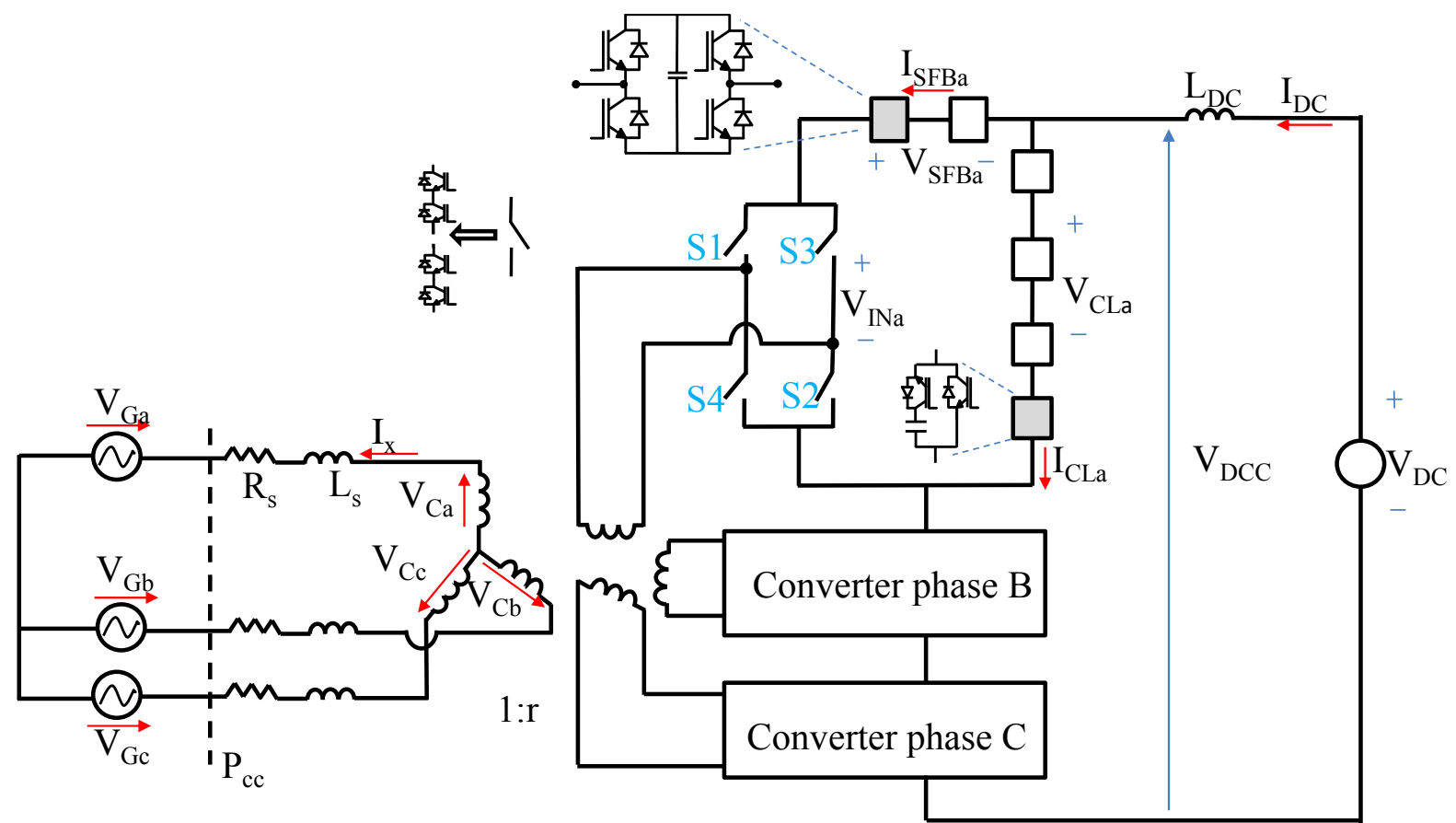

Figure 1: The Series Bridge Converter (SBC) Topology

\section{Operating Principle}

To introduce the basic operating principle of the SBC, consider a balanced and stiff three phase AC network, with the impedances dominated by the leakage inductances of the transformers, and consider first the operation at unity power factor. In this case, ideally, the SFB arms can be considered to be under minimum utilisation. To understand the role of SFBs, assume first that they are all bypassed as discussed in [1]. In this condition, the three CLs generate three full wave rectified sinusoids displaced 
120 electrical degrees. These will be 'unfolded' at the zero-crossings by the main H-bridges, and active power can be exchanged by controlling the phase shift with respect to the grid voltage. The turn ratio $r: 1$ of the transformer can be selected to match the amplitude of the converter voltage and grid voltage in this ideal condition. Consider a generic phase shift $\delta$ between the grid voltage and the converter voltage, and a corresponding phase shift $\varphi$ of the grid current. Consider for instance phase ' $a$ ' in symmetrical AC network, the voltages and currents can be described as (1):

$V_{G a}(t)=V_{G} \sin (\omega t+\delta), \quad V_{C a}(t)=V_{C} \sin (\omega t), \quad I_{a}(t)=I \sin (\omega t+\varphi)$

$V_{I N a}=\left|V_{C a}(t)\right|, \quad V_{S F B a}(t)=0, \quad V_{C L a}(t)=V_{I N a}$

In a balanced three phase system as considered in this research, phase ' $b$ ' and ' $c$ ' voltages and currents are accordingly shifted 120 electrical degrees from each other on the AC side. The full wave rectified sinusoidal voltages formulated by the CLs which are in phase with the respective AC voltages are presented as (2).

$V_{C L a}(t)=V_{C L}|\sin (\omega t-2 y \pi / 3)|$

The average $C L$ voltage, $V_{C L}$ in each phase can be derived (3).

$\bar{V}_{C L}=\frac{2}{\pi} V_{C L} \rightarrow \bar{V}_{D C C}=\sum_{x=a, b, c} \bar{V}_{C L x}=3\left(\frac{2}{\pi} V_{C L}\right) \rightarrow V_{C L}=\frac{\pi}{6} \bar{V}_{D C C}$

Considering Figure 1, the average of the three CL voltages must sum to $\mathrm{V}_{\mathrm{DCC}}$ and therefore the peak $\mathrm{CL}$ voltage is directly related to the DC bus voltage as stated in (3). Also, Fourier spectrum of the individual CL voltages in (2) contains even harmonics $2 \mathrm{n}$, that will cancel in the sum across the three phases except for those harmonics that are also multiples of 3 -i.e. $V_{\text {DCC }}$ will contain $6 n$ ripple components. In the analysis in (2) and (3), the SFB arms are not involved in the voltage formation and the characteristic $6 \mathrm{n}$ harmonics are not eliminated from the DC network, which makes the amplitude of the converter voltages directly related to the DC voltage and influences the selection of the turn ratio of the transformer. To decouple the peak converter voltage, $\mathrm{V}_{\mathrm{C}}$ from the composite $\mathrm{DC}$ voltage in the CLs, the SFB arms would be required to synthesise the residual voltage between the voltage required at the converter terminal, $\mathrm{V}_{\mathrm{Cx}}(\mathrm{t})$ and that synthesised by the corresponding CL arm, $\mathrm{V}_{\mathrm{CLx}}(\mathrm{t})$. Therefore, the SFBs are used to facilitate the reactive power control and improve power quality on the DC side by extracting the $6 \mathrm{n}$ harmonic voltages from the DC circuit. For a given active and reactive power, the amplitude and phase of the converter voltage $\mathrm{VC}<\delta$ are varied with respect to the grid voltage as illustrated in Figure 2 and Figure 3 to active the required power exchange with the grid.

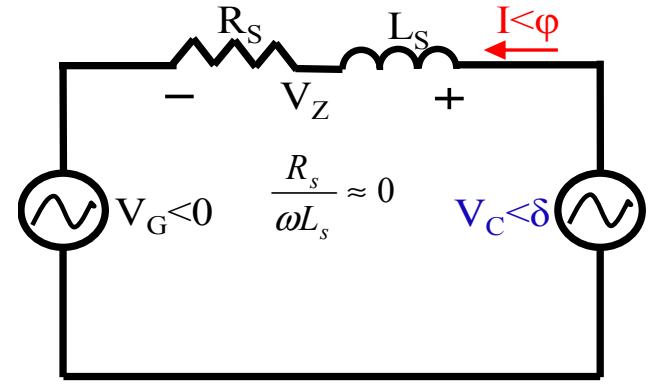

Figure 2: Equivalent AC circuit of Grid connected SBC

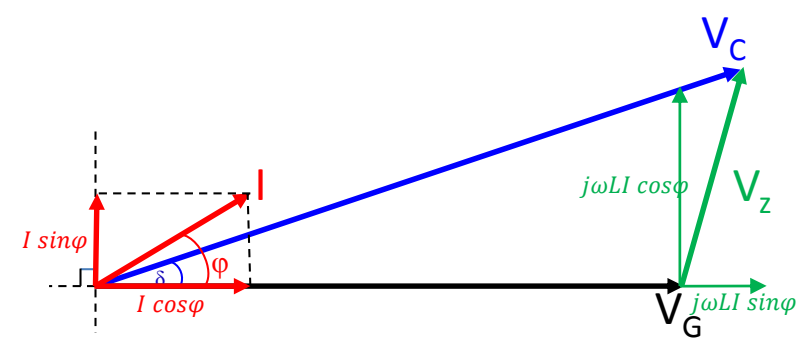

Figure 3: Phasor Diagram

However, as discussed earlier, the amplitude of the rectified sinusoids forming the CL voltages $V_{C L}$ is fixed as shown in (3). The difference between $V_{C L x}(t)(2)$ and $V_{I N x}(4)$ required to generate $V_{C x}(t)$ is managed by the SFBs, guaranteeing active and reactive power control:

$$
V_{I N a}=V_{C}|\sin (\omega t)| \rightarrow V_{S F B a}(t)=\left(V_{C}-V_{C L}\right)|\sin (\omega t)|
$$

In addition, the $\mathrm{DC}$ voltage ripple appearing when the $\mathrm{CL}$ voltages are simply rectified sinusoids, can be subtracted in advance from the CL waveforms - by subtracting one third of the ripple from each CL voltage. To avoid distortion on the AC side, the same ripple must be added to the SFB voltages. From the Fourier analysis of the individual CL voltages in (2) we get (5). 


$$
V_{D C C}=\bar{V}_{D C C}+V_{R}(t) \rightarrow V_{R}(t)=-\frac{12}{\pi} V_{C L} \sum_{n=1}^{\infty}\left(\frac{1}{36 n^{2}-1} \cos (6 n \omega t)\right)
$$

In summary, the CL voltage, the SFB voltage and the input voltage for the main H-bridge that guarantees full power control and DC filtering are (6).

$$
\begin{aligned}
& V_{C L a}(t)=V_{C L}|\sin (\omega t)|-\frac{1}{3} V_{R}(t)=V_{C L}|\sin (\omega t)|+\frac{4}{\pi} V_{C L} \sum_{n=1}^{\infty}\left(\frac{1}{36 n^{2}-1} \cos (6 n \omega t)\right) \\
& V_{S F B a}(t)=\left(V_{C}-V_{C L}\right)|\sin (\omega t)|+\frac{1}{3} V_{R}(t)=\left(V_{C}-V_{C L}\right)|\sin (\omega t)|-\frac{4}{\pi} V_{C L} \sum_{n=1}^{\infty}\left(\frac{1}{36 n^{2}-1} \cos (6 n \omega t)\right) \\
& V_{I N a}(t)=V_{C L a}(t)+V_{S F B a}(t)=V_{C}|\sin (\omega t)| \rightarrow \text { H bridge unfolding } \rightarrow V_{C a}(t)=V_{C} \sin (\omega t)
\end{aligned}
$$

From Figure 1 and (1), the current through the converter arms can be deduced as (7). For sustainable operation of the converter, AC-DC power balance is required. By imposing this constraint, the current through the DC network can be expressed as (8).

$$
\begin{array}{ll}
I_{S F B a}(t)=\operatorname{sgn}\left(V_{C a}(t)\right) I_{a}(t) & I_{C L a}(t)=I_{D C}-I_{S F B}(t) \\
P_{D C}=P_{A C} \rightarrow I_{D C} V_{D C C}=\frac{3}{2} V_{c} I \cos \varphi & I_{D C}=\frac{3 V_{c} I}{2 V_{D C}} \cos \varphi
\end{array}
$$

It can be shown that the average power into the converter CL arm can be expressed as (9) which has a contribution due to the power flow in the DC network and that due to the power exchanged between the converter and $\mathrm{AC}$ network. For all operating points, this power in the CL arm is equal but opposite to that in the SFB arm.

$$
\begin{aligned}
& \bar{P}_{C L a}=\frac{2 V_{C L} I_{D C}}{\pi}-\frac{V_{C L} I}{2} \cos (\varphi)\left(1-\frac{16}{\pi^{2}} \sum_{n=1}^{\infty} \frac{1}{\left(36 n^{2}-1\right)^{2}}\right)=\underbrace{\frac{V_{D C C} I_{D C}}{3}}_{D C \text { Effect }}-\underbrace{K_{U} \frac{V_{C L} I}{2} \cos (\varphi)}_{A C \text { Effect }}=-\bar{P}_{S F B a} \\
& K_{U}=\left(1-\frac{16}{\pi^{2}} \sum_{n=1}^{\infty} \frac{1}{\left(36 n^{2}-1\right)^{2}}\right) \\
& \bar{P}_{C L a}=\frac{V_{D C C} I_{D C}}{3}-K_{U} \frac{V_{C L} I}{2} \cos (\varphi)=-\bar{P}_{S F B a}
\end{aligned}
$$

By substituting the expression for the DC current (8) in the average power (9), it can be shown that two conditions are required for the power in the arms to be equal and zero. i.e $\mathrm{K}_{\mathrm{U}}=1$ and $\mathrm{V}_{\mathrm{CL}}=\mathrm{V}_{\mathrm{C}}$ or $\mathrm{P}_{\mathrm{DC}}=\mathrm{P}_{\mathrm{AC}}=0$. Generally $\bar{P}_{C L a}=-\bar{P}_{S F B a} \neq 0$ and without an active energy management inter-arm energy control would not be possible.

\section{Inter Arm Energy Management}

As discussed in Section III, the proposed method of voltage and current formation in the SBC optimises the use of the converter arms and results in a compact configuration. However, the power flow analysis shows that the net power in the arms is not naturally zero and active control measures are required to re-establish the energy balance guaranteeing operability and the practical feasibility of the converter.

To overcome this limitation, a means of "moving" power from the CL to the SFB and vice versa is needed. The solution proposed here is to exploit a simple observation: it can be seen from Figure 1 and equation (7) that both CL and SFB currents have the same AC components, dominated by the $2^{\text {nd }}$ harmonics of the line frequency. By adding to the CL and SFB voltages two $2^{\text {nd }}$ harmonic voltages in phase opposition with each other, these will contribute to the total $\bar{P}_{C L a}$ and $\bar{P}_{S F B a}$ with the same power but with opposite sign, enabling power cancellation and achieving the balance condition $\bar{P}_{C L a}=-\bar{P}_{S F B a}=0$. It is important to observe that the three $2^{\text {nd }}$ harmonic voltages added to the CL voltages do not affect the DC filtering, as they cancel over the three phases. Similarly, they do not affect the voltages $\mathrm{V}_{\mathrm{INx}}$ at the input of the main H-bridges, as they are added to CLs and SFBs with opposite signs. 
Now consider a system where the converter CL and SFB voltages are redefined with a proportion of second harmonic voltage as (11).

$$
\begin{aligned}
& V_{C L a}(t)=V_{C L}|\sin (\omega t)|-V_{R}(t)+V_{E M a}(t) \\
& V_{S F B a}(t)=\left(V_{C}-V_{C L}\right)|\sin (\omega t)|+V_{R}(t)-V_{E M a}(t) \\
& V_{E M a}(t)=-\frac{4 K V_{C L}}{3 \pi} \cos (2 \omega t+\gamma)
\end{aligned}
$$

$\mathrm{K}$ is the proportion of second harmonic voltage in an arm to be injected for energy management. The net active power in the converter arms can now be derived as (12).

$$
\bar{P}_{C L a}=\frac{V_{D C C} I_{D C}}{3}-K_{U} \frac{V_{C L} I}{2} \cos (\varphi)+K_{E M} \frac{V_{C L} I}{2}=-\bar{P}_{S F B a}
$$

Clearly, the extra degree of freedom in $\mathrm{K}_{\mathrm{EM}}$ can be used to move power between the two arms in a phase. This concept presents a novel method of inter arm energy management control ensuring controllability of the energy in the converter arms.

\section{General Converter Control}

A method of waveform formation in the SBC which optimises the size of the converter arms while achieving active and reactive power flow controllability has been introduced. As confirmed with the analysis in Section IV, the concept requires an active control scheme to redistribute power between the arms in a phase and achieve inter arm balancing as discussed in Section IV. Clearly with these methods of voltage formation and inter arm energy management, the operation of the converter is well sustained for all operating points within the designed operating envelope. However, in practice appropriate control schemes are required to manage the power exchange between the various parts of the HVDC system and to ensure the effects of dynamic influences are well controlled. The general control structure adopted to ensure sustainable operation of the converter is illustrated in Figure 4.

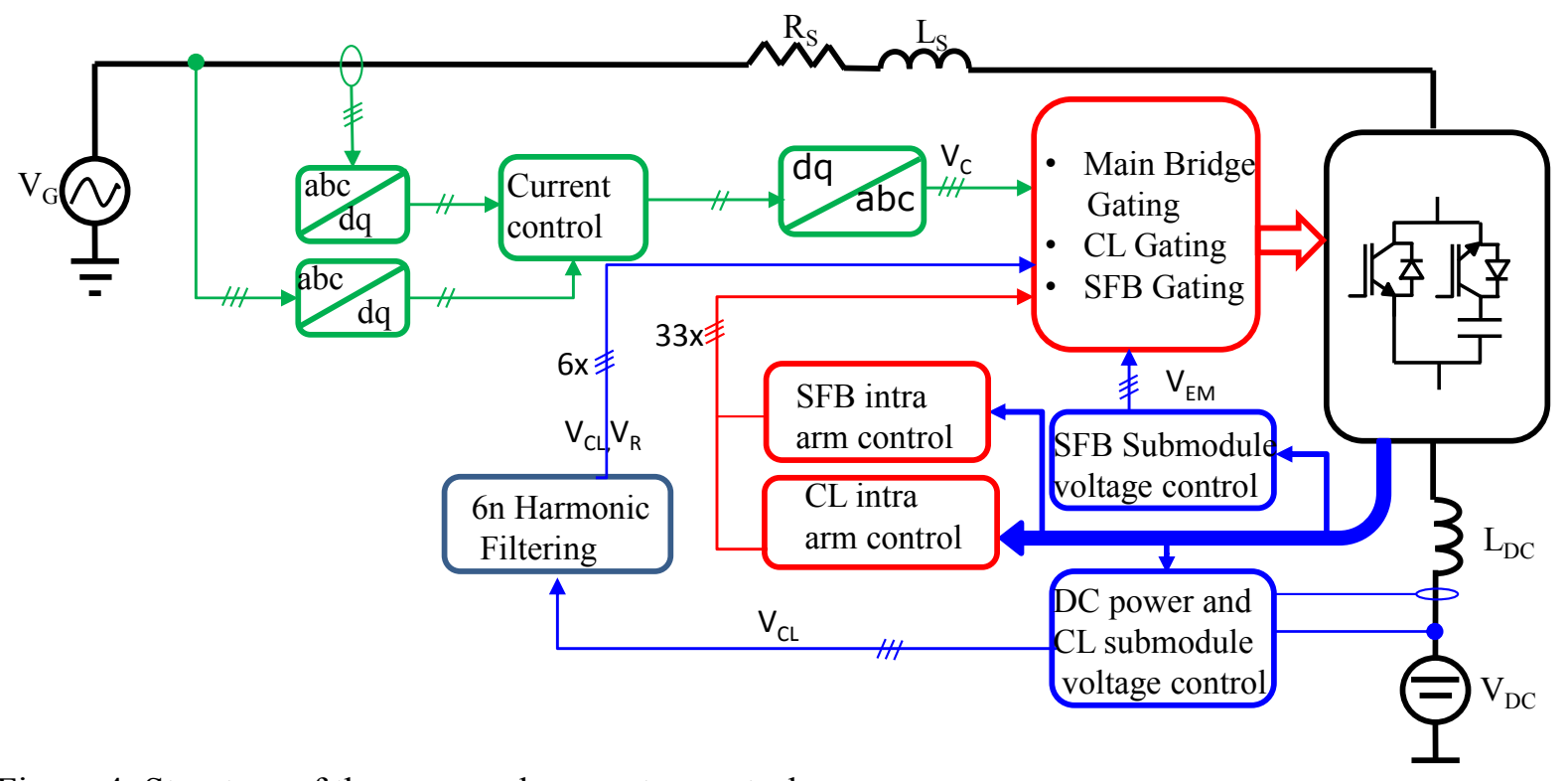

Figure 4: Structure of the proposed converter control

The control scheme can be divided in four main parts:

AC side control

DC Side Control

Inter arm energy control

Intra arm energy control

In the $\mathrm{AC}$ side control, standard vector current control is used to manage the power exchanged between the $\mathrm{SBC}$ and the $\mathrm{AC}$ network. In this system the $\mathrm{AC}$ system voltages and currents are measured and transformed to DC quantities where linear control methods (in this case Proportional 
plus Integral $(\mathrm{P}+\mathrm{I}))$ can be applied to manage the power flow. The output of the AC control also presents the required converter voltages which are used to synchronise the CL and SFB voltage waveforms.

The DC side controller is also a linear control scheme that acts to ensure the active power of the converter on the $\mathrm{AC}$ side is matched by that in the $\mathrm{DC}$ network. This controller also manages the total energy within the converter, ensuring that the total submodule capacitor voltages are maintained. As discussed earlier, maintaining the total energy within the converter does not automatically guarantee power balance between the two arms in a converter phase. The inter arm energy control - in the form of SFB submodule voltage control derives the amount of second harmonic voltage, $\mathrm{V}_{\mathrm{EM}}$ to be injected into the converter arms to maintain the energy level within the arms.

The intra arm energy management control is a passive control scheme based on sorting algorithm to re-assign the submodules to different virtual positions within the chain of submodules. The algorithm uses a quick sort algorithm to optimise the performance of the control and reduce the execution time. The implementation of the intra-arm control ensures that over time, the energy within the converter arm is equally distributed among the submodules. Generally, the algorithm presents sorting indices for each submodule which is used in conjunction with the required arm voltage to generate the gating signal for the devices in each submodule.

\section{Simulation Results}

This section presents selected waveforms illustrating the concept of converter operation as discussed in Sections III and inter-arm energy management principle presented in Section IV. The results have been obtained from both an average model - illustrating the performance of the converter with an infinite number of submodules and a switching model of a medium voltage scaled demonstrator. Parameters of the system modelled in this study to validate the concepts are presented in Table 1.

Table 1: Parameters of the Modelled System

\begin{tabular}{|l|l||}
\hline System Parameter & Value \\
\hline Rated Active power, $\mathrm{P}$ & $\pm 20 \mathrm{MW}$ \\
Rated Reactive power, Q & $-6.6 \mathrm{MVAr} / 8.2 \mathrm{MVAr}$ \\
AC System Voltage, $\mathrm{V}_{\mathrm{G}}$ & $11 \mathrm{kV}$ \\
AC Equivalent Inductance, $\mathrm{L}_{\mathrm{s}}$ & $2.3 \mathrm{mH}$ \\
DC Bus Voltage, $\mathrm{V}_{\mathrm{DC}}$ & $20 \mathrm{kV}$ \\
DC Link Inductance, $\mathrm{L}_{\mathrm{DC}}$ & $10 \mathrm{mH}$ \\
Submodule Capacitance, C & $4 \mathrm{mF}$ \\
Number of submodules in CL, $\mathrm{n}_{\mathrm{CL}}$ & 8 \\
Number of submodules in SFB, $\mathrm{n}_{\mathrm{SFB}}$ & 3 \\
\hline \hline
\end{tabular}

The waveforms from an average model of the SBC in MATLAB/PLECS simulation of a medium voltage demonstrator rated for $\mathrm{V}_{\mathrm{DC}}=20 \mathrm{kV}, \mathrm{V}_{\mathrm{AC}}=11 \mathrm{kV}$ line to line and $\mathrm{PQ}$ operating envelope between $\pm 20 \mathrm{MW}$ and 8.2MVAr capacitive and 6.6MVAr inductive clearly show the high quality waveforms that can be achieved with an infinite number of submodules. For the sake of simplicity, all the waveforms are referred to the converter side of the transformer, and presented according to the sign conventions depicted in Figure 1. Figure 5 shows the main converter waveforms when operating at $\mathrm{P}=20 \mathrm{MW}, \mathrm{Q}=0 \mathrm{MVAr}$. The CL and SFB voltages include $\mathrm{DC}$ ripple cancellation and $2^{\text {nd }}$ harmonic voltage for inter arm energy management. From the results in Figure 5, it can be observed that the DC bus voltage is ripple free, and the SFBs have minimal contribution in the converter voltage formation. Figure 6 presents instantaneous and average CL powers (average SFB power are equal but with opposite sign) for phase ' $a$ ' with and without 2 nd harmonic voltage injection for inter arm energy management, confirming the effectiveness of the proposed energy management scheme. 
(a)

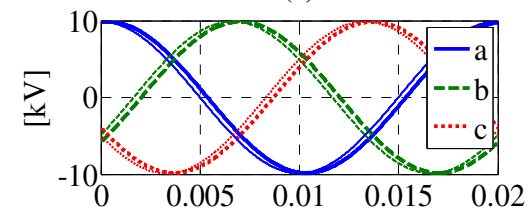

(b)

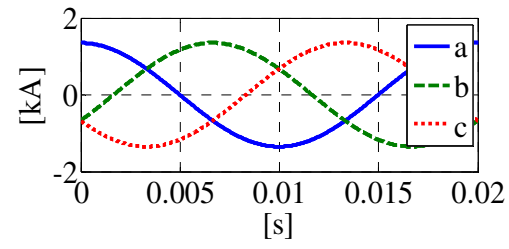

(c)

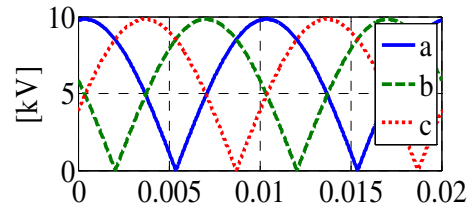

(d)

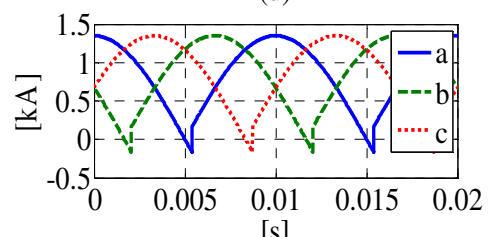

(e)

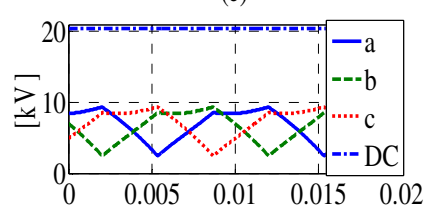

(f)

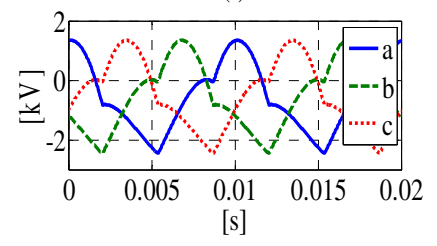

Figure 5: Waveforms at $\mathrm{P}=20 \mathrm{MW}, \mathrm{Q}=0 \mathrm{MVar}$ with $6 \mathrm{n}$ ripple cancellation and inter arm energy management - (a) Grid voltages $\mathrm{V}_{\mathrm{Gx}}$ (thin lines) and converter voltages $\mathrm{V}_{\mathrm{Cx}}$ (thick lines) (b) Grid currents (c) Main H-bridge voltages $\mathrm{V}_{\text {Inx }}$ (d) Folded currents $\mathrm{I}_{\mathrm{SFBx}}$ (e) CL voltages (f) SFB voltages

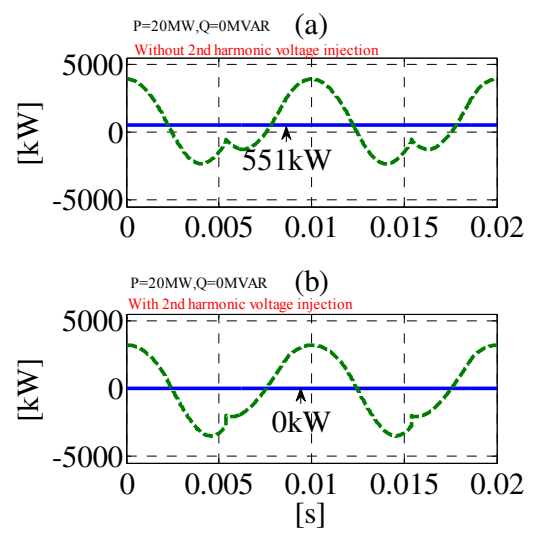

Figure 6: $\mathrm{CL}$ instantaneous (dashed) and average powers (a) $\mathrm{P}=20 \mathrm{MW}, \mathrm{Q}=0 \mathrm{MVar}$ without 2 nd harmonic injection, (b) with 2nd harmonic injection
The steady state results presented in Figure 5 and 6 validating the theoretical concept of converter operation are further validated in a similar scaled switching model of the SBC converter. Considering the voltage range requirement of the CL and the SFB arms, 8 half bridge submodules and 3 full bridge modules rated at a nominal voltage of $1.5 \mathrm{kV}$ with a local capacitor of $4 \mathrm{mF}$ each are used to populate the converter. To compensate for the smaller number of submodules used in the demonstrator compared to that which would be required in a practical system, a PWM method is used to modulate the submodules to increase the voltage fidelity and waveform quality. However, the 'main' H-bridge is still switched at the fundamental frequency. The converter is operated to exchange active and reactive power with the grid as would be expected of a VSC-HVDC converter. In line with the results presented in Figure 5 and 6 , further results from an actual switching model are presented to support the operation of the converter within the designed operating envelope.

The voltages synthesised by the CL arms, the total across the three CL arms and that synthesised by the SFB arms are presented in Figure 7a. It can be observed that the total CL voltage, $\mathrm{V}_{\mathrm{DCC}}$, is free of the low order harmonics other than high frequency switching effects. Clearly, these high frequency switching harmonics will be absent from any practical high voltage high power implementation with hundreds of submodules. This observation is evident in the average model waveforms representing a system with an infinite number of submodules, where the total CL voltage is free of any harmonic components. As mentioned earlier, the voltage demand on the SFB arm is very small compared to that required by the CL arms. Internal energy management control is confirmed with the successful control of the CL and SFB submodule capacitor voltages around the nominal value of $1.5 \mathrm{kV}$ in Figure $7 \mathrm{~b}$. The peak-peak ripple of the submodule capacitor voltages is about $12 \%$ for the CL cells and $8.5 \%$ for the SFBs cells at unity PF operation. 

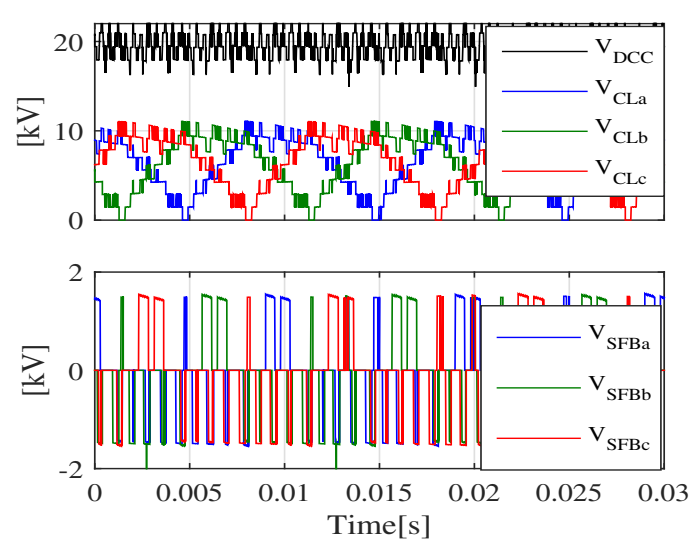

(a) CL (upper plot) and SFB (lower plot) arm voltages
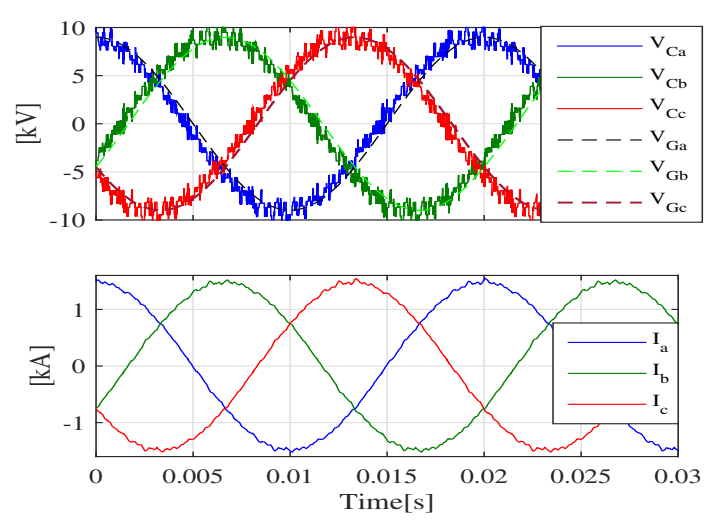

(c) Converter and Grid voltages (upper plot) and line currents (lower plot)
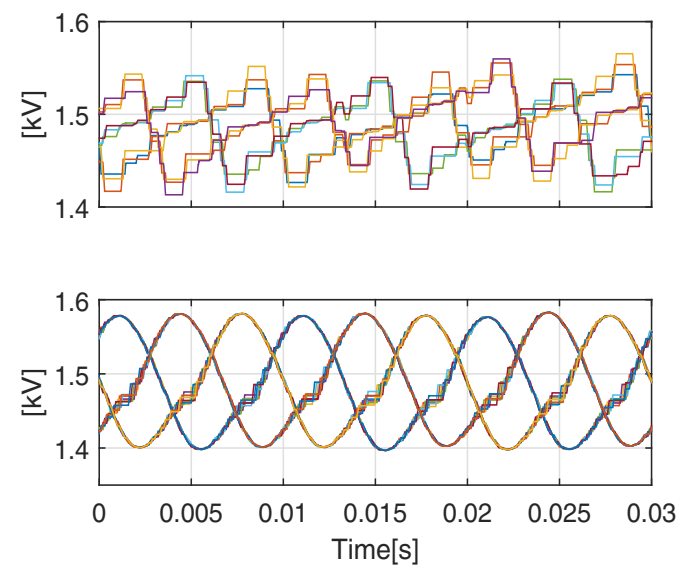

(b) CL (lower plot) and SFB (upper plot) submodule capacitor voltages
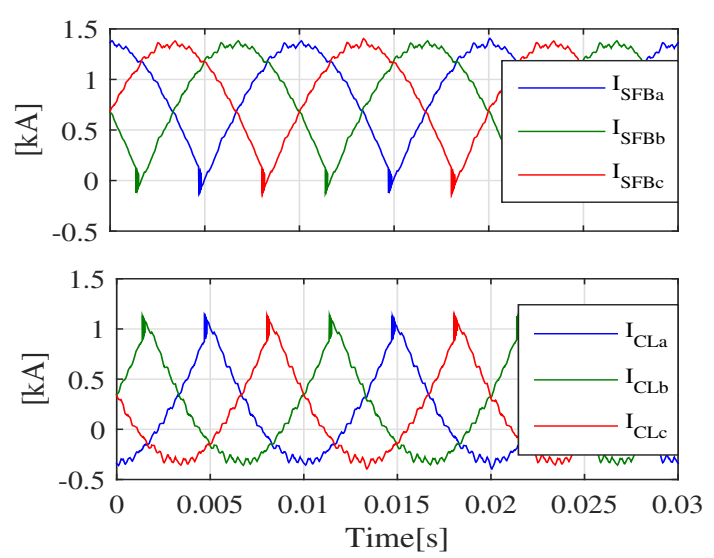

(d) SFB currents (upper plot) and CL currents (lower plot)

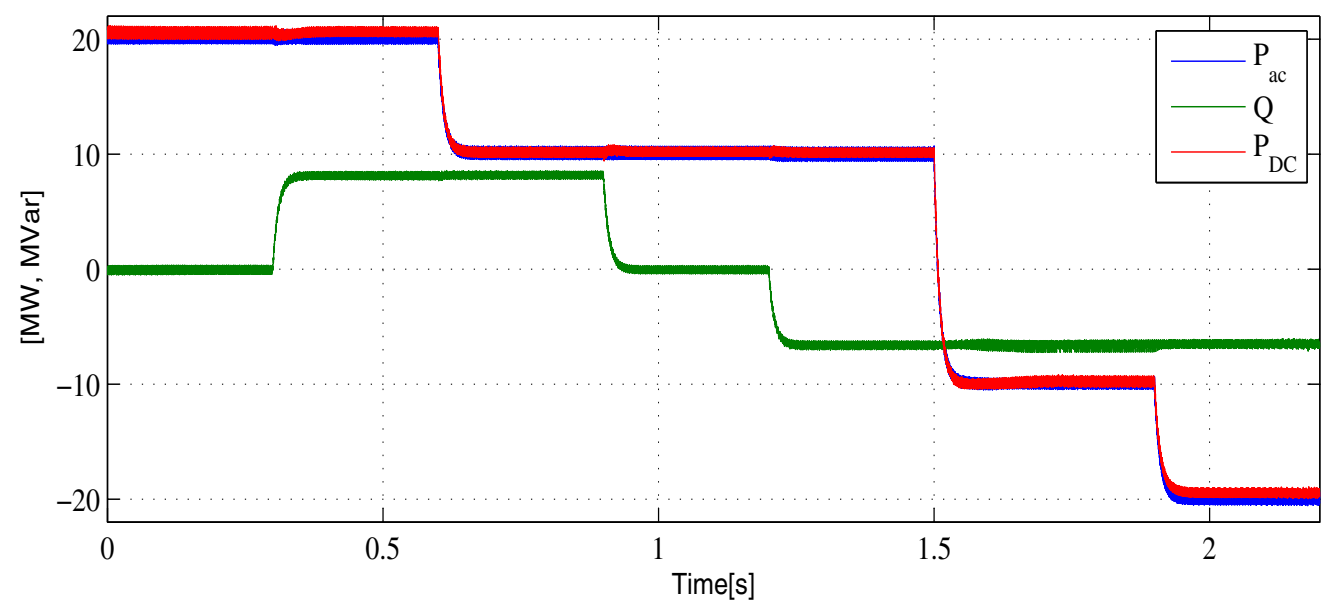

(e) Power traversal through the design operating envelope: - Rated active power (Inverting) $\rightarrow$ rated active (Inverting) and reactive power (capacitive) $\rightarrow$ half rated active (Inverting) and full reactive power (capacitive) $\rightarrow$ half rated active power (Inverting) $\rightarrow$ half rated active and full reactive power (inductive) $\rightarrow$ half rated active (Rectifying) and full reactive power (inductive) $\rightarrow$ rated active (Rectifying) and full reactive power (inductive)

Figure 7: Plots illustrating the converter operating principle and power traversal through different operating points 
It can be observed from Figure $7 \mathrm{c}$, that the voltage synthesised by the converter and the current exchanged between the converter and the grid are of high quality. The converter voltage leads the grid voltage, as you would expect when the converter exports power to the grid. In Figure 4d, the current through the arms of the converter are presented. As expected from the initial analysis, the currents in both arms of a phase have the same AC components, but with opposite phase In Figure 4e, transient performance of the converter is demonstrated with power traversal from rated power (Unity PF) through rated power and full reactive power (capacitive) to rated power and full reactive power inductive. In all this power traversal, internal energy management of the converter is maintained, ensuring the converter operation is sustainable.

\section{Conclusions}

In this paper, the SBC converter has been proposed which has the advantage of using significantly fewer submodules than other topologies with similar performance characteristics. The proposed converter uses an optimum number of half bridge and full bridge submodules to achieve active and reactive power control and DC active filtering. The operating principle of the converter, the voltage waves shaping and internal energy management principle have been discussed. Results from a scaled down medium voltage demonstrator have been presented to validate the concept of converter operation and the capabilities of the converter.

\section{REFERENCES}

[1] R. Feldman, M. Tomasini, E. Amankwah, J. C. Clare, P. W. Wheeler, D. R. Trainer, et al., "A Hybrid Modular Multilevel Voltage Source Converter for HVDC Power Transmission," Industry Applications, IEEE Transactions on, vol. 49, pp. 1577-1588, 2013.

[2] S. Allebrod, R. Hamerski, and R. Marquardt, "New transformerless, scalable Modular Multilevel Converters for HVDC-transmission," in Power Electronics Specialists Conference, 2008. PESC 2008. IEEE, 2008, pp. 174-179.

[3] M. M. C. Merlin, T. C. Green, P. D. Mitcheson, D. R. Trainer, R. Critchley, W. Crookes, et al., "The Alternate Arm Converter: A New Hybrid Multilevel Converter With DC-Fault Blocking Capability," Power Delivery, IEEE Transactions on, vol. 29, pp. 310-317, 2014.

[4] G. P. Adam, Finney, S. J., Williams, B. W.,Trainer, D. R.,Oates, C. D. M.,Critchley, D. R., "Network fault tolerant voltage-source-converters for high-voltage applications," in $A C$ and DC Power Transmission, 2010. ACDC. 9th IET International Conference on, 2010, pp. 1-5.

[5] G. Bopparaju, "VSC based FACTS and HVDC: ABB's experience," in Sustainable Energy and Intelligent Systems (SEISCON 2011), International Conference on, 2011, pp. I-2-I-2.

[6] L. B. Feng Wang, Tuan Le, "An Overview of VSC-HVDC : State - of - art and potential applications in Electric Power Systems," Cigre, 2011.

[7] J D Ainsworth, M Davies, P J Fitz, K E Owen, and D. R. Trainer, "A static var compensator (STATCOM) based on single phase chain circuit convertors," IEE Proceedings, Generation, Transmission and Distribution, vol. 145, July 1998.

[8] A. Lesnicar and R. Marquardt, "An innovative modular multilevel converter topology suitable for a wide power range," in Power Tech Conference Proceedings, 2003 IEEE Bologna, 2003, p. 6 pp. Vol.3.

[9] K. Friedrich, "Modern HVDC PLUS application of VSC in Modular Multilevel Converter topology," in Industrial Electronics (ISIE), 2010 IEEE International Symposium on, 2010, pp. 3807-3810.

[10] D. R. Trainer, Davidson, C. C., Oates, C. D. M., Macleod, N. M., \& Critchley, D. R., "A New Hybrid Voltage-Sourced Converter for HVDC Power Transmission," in Cigre 2010.

[11] E. K. Amankwah, J. C. Clare, P. W. Wheeler, and A. J. Watson, "Cell capacitor voltage control in a parallel hybrid modular multilevel voltage source converter for HVDC applications," in Power Electronics, Machines and Drives (PEMD 2012), 6th IET International Conference on, 2012, pp. 1-6.

[12] O. F. Jasim and D. R. Trainer, "Voltage Source Converter," European Patent Application EP2858231A1, 8 ${ }^{\text {th }}$ April, 2015. 